\title{
EXACT NONLINEAR SOLUTION FOR CONSTANT-RATE EXPRESSION FROM MATERIAL OF FINITE THICKNESS
}

\author{
P. BROADBRIDGE ${ }^{1,2}$ and P. J. BANKS ${ }^{3}$
}

(Received 10 December 1990; revised 28 March 1991)

\begin{abstract}
We present new exact solutions for the flow of liquid during constant-rate expression from a finite thickness of liquid-saturated porous material with nonlinear properties. By varying a single nonlinearity parameter and a dimensionless expression rate, we systematically investigate the effect of nonlinearity and of an impermeable barrier (e.g. a piston). We illustrate the water profile shape and the water ratio deficit at the expression surface (e.g. a filter membrane) as a function of time.
\end{abstract}

\section{Introduction}

It has proved to be a difficult task to predict, analytically, the external pressure required to maintain constant-rate expression of liquid from a porous material undergoing steady piston compression. The practical importance of this problem has been discussed by Banks [1] and by Banks and Burton [2]. Here, we consider a finite undimensional prism of a liquid-saturated porous material bounded at one end by a permeable membrane and at the other end by an impermeable piston. If we were able to predict the water content at the membrane, then this would yield the applied external pressure via the nonlinear stress-strain relationship for the porous material. However, the water content at one particular location could be predicted only by solving the nonlinear equation for flow throughout the entire material depth. It has

\footnotetext{
${ }^{1}$ Department of Mathematics, La Trobe University, Bundoora, Vic. 3083, Australia.

${ }^{2}$ Present address: Department of Mathematics, University of Wollongong, Wollongong, NSW 2500, Australia.

${ }^{3}$ CSIRO Div. of Building, Construction and Eng., P.O. Box 56, Highett, Vic. 3190, Australia.

(C) Copyright Australian Mathematical Society 1992, Serial-fee code 0334-2700/92
} 
been shown that, at least for very wet slurries, the distribution of water may be predicted well even after neglecting both side-wall friction and irreversible rheological properties of the solid-liquid mixture [20]. After neglecting both of these complications, as shown previously [1], the liquid flow may be modelled by a nonlinear diffusion equation

$$
\frac{\partial e(m, t)}{\partial t}=\frac{\partial}{\partial m}\left[E(e) \frac{\partial e}{\partial m}\right]
$$

subject to uniform initial conditions

$$
e=e_{i} \text { (constant) }, \quad t=0,0 \leq m \leq M,
$$

constant-rate expression at the membrane boundary,

$$
E(e) \frac{\partial e}{\partial m}=q(\text { constant }), \quad m=0, t>0,
$$

and no flow at the piston boundary,

$$
\frac{\partial e}{\partial m}=0, \quad m=M, t>0
$$

As defined previously [1],

$e$ is the void ratio (equal to water ratio, in saturated materials),

$m$ is a material distance coordinate,

$t$ is the time,

$E$ is the expression coefficient (or Lagrangian liquid diffusivity [20]),

$q$ is the expression rate, and

$M$ is the total volume of solid component per unit cross section area.

The same description would apply if the piston as well as the membrane were permeable. In that case, the position $m=M$ of no flow would represent the midpoint between the two boundaries.

Solutions of the linear (constant- $E$ ) model, presented in [1] and [2], may approximate the liquid flow in a limited class of materials, but for many other materials the expression coefficient depends strongly on the void ratio $e$, so that the flow equation (1) and the boundary condition (3) are both highly nonlinear. Here, we solve analytically a nonlinear liquid-flow model. We examine systematically the effect of the degree of nonlinearity on the flow pattern, by varying a single parameter of the model. The crucial value of the membrane water ratio, at any particular time, may be obtained directly from the analytic solution, without first obtaining preliminary estimates of the flow profile after many successive time increments, which is a necessary disadvantage of approximate numerical solution schemes. Previously, analytic and approximate analytic solutions ([4], [19] and [20]) have neglected 
finite-column end effects. These earlier semi-infinite column solutions apply to constant-rate expression only in the early stage, before the water ratio begins to reduce significantly near the piston.

\section{The exactly-solvable model}

We assume that the expression coefficient has been represented by a function of the type

$$
E=\gamma /(b-e)^{2},
$$

with $\gamma$ and $b$ constant. We assume that the representation (5) holds in a range of the void ratio $e_{i} \geq e \geq e_{f}$, where $e_{i}$ is the initial water ratio and $e_{f}$ is a minimum water ratio. We define the normalized void ratio

$$
g=\left(e-e_{f}\right) /\left(e_{i}-e_{f}\right)
$$

and the dimensionless expression coefficient $E_{*}(g)=E(e) / \bar{E}$, where $\bar{E}$ is the arithmetic mean,

$$
\bar{E}=\frac{1}{\left(e_{i}-e_{f}\right)} \int_{e_{f}}^{e_{i}} E(e) d e .
$$

For the model (5), we have

$$
\bar{E}=\frac{\gamma}{c(c-1)\left(e_{i}-e_{f}\right)^{2}},
$$

where $c=\left(b-e_{f}\right) /\left(e_{i}-e_{f}\right)$.

Hence,

$$
E_{*}(g)=c(c-1) /(c-g)^{2} .
$$

With $g$ always normalised to vary between 0 and 1 , the parameter $c$ provides a comparison of the degree of nonlinearity of different materials over possibly different ranges of water ratio. For highly nonlinear materials, $c$ is close to one.

The two independent parameters in (5) may be taken to be the nonlinearity parameter $c$ and the sorptivity $S$ of Philip [19], a convenient choice since the latter may be measured directly in a constant-pressure experiment. In a nonlinear diffusion (1) in a semi-infinite column subject to uniform initial condition (2) and constant-concentration boundary condition

$$
e=e_{f}, \quad m=0,
$$

(which, in the context of expression, is equivalent to a constant-pressure condition), the depth of the separated liquid will be

$$
l=S\left(e_{i}, e_{f}\right) t^{1 / 2} \text {. }
$$


In general heterogeneous media, at sufficiently early times, (10) remains valid [3] (neglecting higher order corrections) when $S$ refers to the local environment at $m=0$. In particular, at sufficiently early times, the effect of a finite macroscopic column length will be negligible and $S$ may be measured directly. The parameter $\gamma$ of (5) may be expressed, in terms of $c$ and $S$, as

$$
\gamma=h_{-}(c) S^{2} \text {, }
$$

where $h_{-}$is a function of $c$ alone. The function $h_{-}(c)$, appropriate for an increasing expression coefficient $E_{*}(g)$, is different from the function $h(c)$ of Broadbridge and White [6] which applies in the case of decreasing $E_{*}(g)$. From the analytic solution of Fujita [9], which satisfies the boundary condition (9), we obtain the one-to-one relation

$$
\frac{1}{c-1}=\frac{1}{2} \sqrt{\pi} h_{-}^{-\frac{1}{2}} \operatorname{erf} c\left(-\frac{1}{2} h_{-}^{-\frac{1}{2}}\right) \exp \left(\frac{1}{4} h_{-}^{-1}\right) .
$$

From (7) and (11), the mean expression coefficient may be expressed in terms of $c$ and $S$, as

$$
\bar{E}=\frac{h_{-}(c)}{c(c-1)} \frac{S^{2}}{\left(e_{i}-e_{f}\right)^{2}} .
$$

If the nonlinearity parameter $c$ is close to 1 , the dimensionless expression coefficient $E_{*}$ depends strongly on normalised void ratio $g$, whereas $E_{*}$ remains close to 1 if $c$ is much larger than 1 . In Figure 1 (page 434), we show least-squares fits of (5) to data from two materials. The first is a sample of asmined Loy Yang brown coal [2], and the second is a filter cake formed from ground and slurried brown coal from the same location [17]. The filter cake from ground coal is seen to be a weakly nonlinear material, with $c=2.07$. The as-mined coal is a moderately nonlinear material, with $c=1.15$. However, for the latter material, as $g$ approaches $0, E_{*}$ decreases more rapidly than the inverse square law model (5) would indicate. As a consequence of the analytic model being only an approximation to real materials, the fitted value of $c$ will in practice depend on the range of $e$ considered.

\section{Exact solutions of the nonlinear model}

Full technical details of the method of solution are given in the Appendix. Here, we outline the new features of this solution.

In the special case that the expression coefficient $E(e)$ takes the form (5), the nonlinear diffusion equation (1) may be reduced to a linear convectiondiffusion equation by a nonlinear transformation of both the dependent (concentration) variable and the independent spatial coordinate. For the case of 


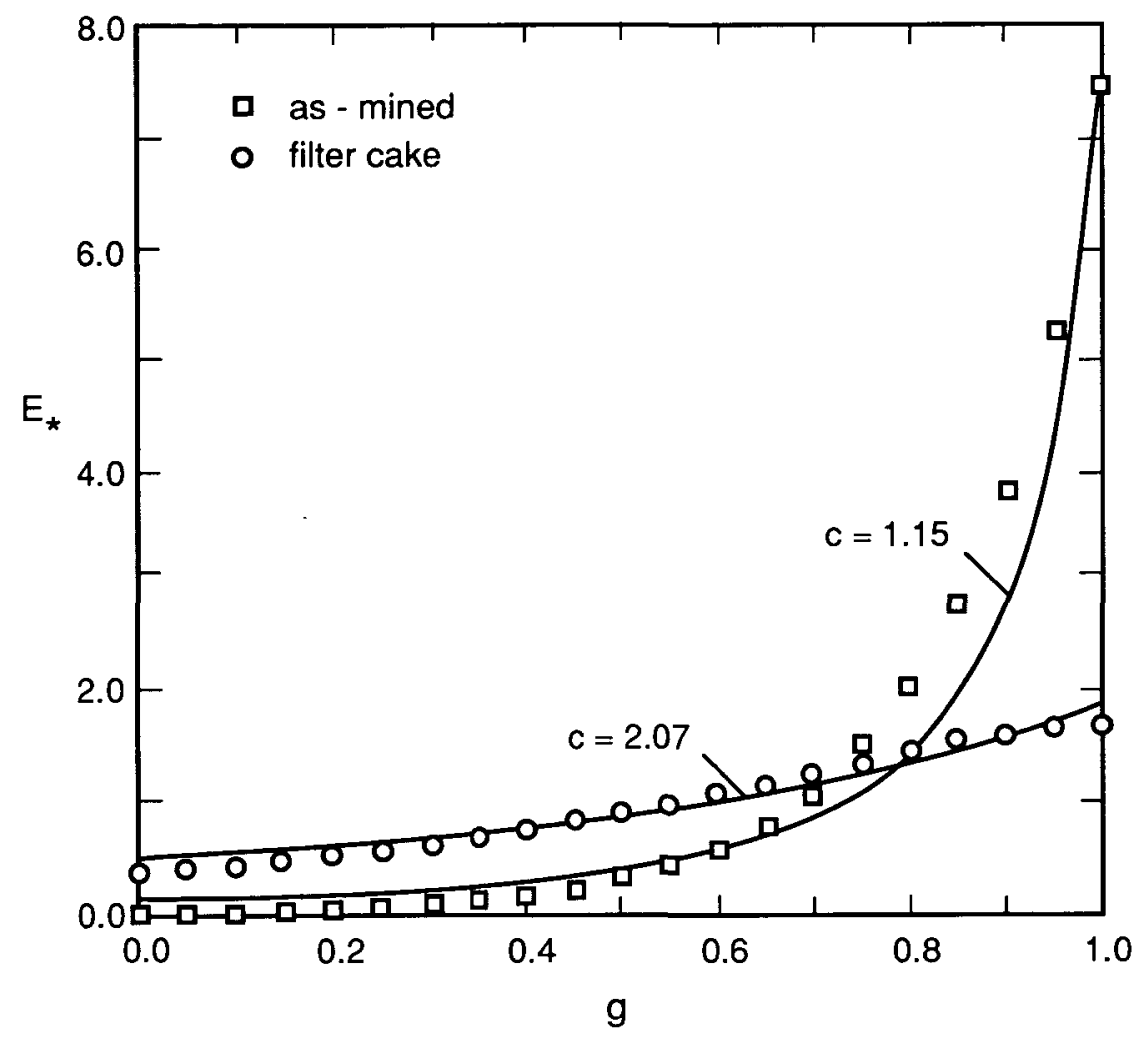

FIGURE 1. Experimental values of dimensionless expression coefficient $E_{*}$ plotted against normalised water ratio $g$, for a sample of as-mined Loy Yang coal $\left(e_{i}=2.34, e_{f}=0.67\right.$, $\left.\bar{E}=9.7 \times 10^{-8} \mathrm{~m}^{2} \mathrm{~s}^{-1}\right)[2]$, and for a sample of filter cake from ground Loy Yang coal $\left(e_{i}=\right.$ $3.11, e_{f}=0.25, \bar{E}=3.8 \times 10^{-5} \mathrm{~m}^{2} \mathrm{~s}^{-1}$ ) [17]. The lines are the respective least squares fits of the model (8).

an idealised semi-infinite region $(M=\infty)$, an exact solution, satisfying a constant-concentration boundary condition, was obtained by Fujita [9]. The exact solution on a semi-infinite domain, satisfying a constant-flux boundary condition, was given by Knight and Philip [14].

After a simple transformation $\tilde{e}=1-e$ of the dependent variable, the analytic solution for the supply of liquid at constant rate, in a medium with increasing expression coefficient $E(e)$, becomes a solution for extraction of liquid at constant rate, from a medium with decreasing expression coefficient $\widetilde{E}(\tilde{e})=E(e)$. This analytic solution agrees very well with water content profiles observed experimentally in very wet slurries, at least until water begins to deplete significantly at the impervious boundary [4]. At this later stage, finite-column end effects will become important and the semi-infinite 
domain model will cease to be applicable. So far, the finite-column impervious boundary condition (4) has defied exact analysis. Following the usual change of independent variables [14] which is used to transform (1) to a linear equation, because of the time dependence of the new spatial coordinate, the two transformed boundaries are in uniform relative motion. Thus, we may transform the nonlinear diffusion problem on a fixed finite region to a linear diffusion problem on a uniformly expanding region. Boundary conditions on an expanding medium cannot be treated algebraically by standard Laplacetransform techniques. However, one key to unlocking this class of problems has been provided by King's theory of Laplace transform boosts [12]. The boundary conditions may be treated by solving difference equations, rather than algebraic equations. So far, this new technique has been applied to nonlinear diffusion-convection problems on finite domains [12, 5]. However, it is not a trivial matter to obtain a limiting solution for the case of zero convection and pure nonlinear diffusion, as the latter problem is quite different in character. The pure nonlinear diffusion problem is equivalent to a linear convection-diffusion problem with one linear radiation boundary condition, whereas after transforming the nonlinear diffusion-convection problem, the radiation boundary condition is replaced by a simpler concentration boundary condition [12].

Another difference between the model developed here, and its related precedents, is that we concentrate here on expression from media for which $E(e)$ is an increasing function. By general arguments [4], this is expected to be the case in moderately wet saturated porous media, as opposed to very wet slurries, in which $E(e)$ is usually decreasing. Although a model expression coefficient (5) may represent either a decreasing function or an increasing function, the analytic solutions require distinct mathematical techniques in the two cases. For constant-rate extraction with $E(e)$ decreasing, or for constant-rate supply with $E(e)$ increasing, the nonlinear diffusion problem transforms to a linear convection-diffusion problem on a uniformly shrinking domain. In transformed coordinates, the domain shrinks to nothing in some finite time $t_{\max }$. In the nonlinear convection-diffusion problem solved so far $[12,5], t_{\max }$ is the radius of convergence of a series solution. This is sufficient for practical purposes, since $t_{\max }$ is larger than the time required either to remove all initial liquid or to fill all initially unoccupied pores. In the class of constant-rate extraction problems with $E(e)$ increasing, considered here, or for constant-rate supply problems with $E(e)$ decreasing, the nonlinear diffusion problem transforms to a linear convection-diffusion problem on an expanding, rather than shrinking medium. The series solution, obtained here, converges for all $t$, even though for our particular purposes we do not require a solution beyond the time for the minimum value of $e$ to reach $e_{f}$. 
In terms of dimensionless variables, the nonlinear diffusion equation (1) becomes

$$
\frac{\partial g}{\partial T}=\frac{\partial}{\partial \xi}\left[E_{*}(g) \frac{\partial g}{\partial \xi}\right],
$$

where $E_{*}(g)=\frac{c(c-1)}{(c-g)^{2}}, g=\frac{e-e_{f}}{e_{i}-e_{f}}, \xi=m / M$ and $T=\bar{E} t / M^{2}$. The boundary and initial conditions are

$$
E_{*}(g) \partial g / \partial \xi=R, \quad \xi=0 .
$$

where $R=M q /\left(e_{i}-e_{f}\right) \bar{E}$,

$$
\partial g / \partial \xi=0, \quad \xi=1,
$$

and

$$
g=1, \quad T=0 .
$$

The exact parametric solution of the boundary-value problem (14)-(17) is given in (A41), (A42) and (A48) of the Appendix. In Figure 2a, we compare the exact water ratio profile in a weakly nonlinear model $(c=2.0)$ to that in a linear model $(c=\infty)$, during expression at constant rate $R=0.5$. Profiles are plotted at times for which $R T=0.2,0.35$ and 0.5 . The variable $R T$ is closely related to the time, to the mean strain $\bar{u}$ and to the mean normalised water ratio $\bar{g}$, as follows:

$$
R T=\frac{q t}{\left(e_{i}-e_{f}\right) M}=\bar{u} \frac{1+e_{i}}{e_{i}-e_{f}}=1-\bar{g} .
$$

At the membrane boundary $\xi=0$, the gradient $\partial g / \partial \xi$ of the water ratio profile is given by

$$
\begin{aligned}
\frac{\partial g}{\partial \xi} & =R / E_{*}(g) \\
& =\frac{R[c-g(0, T)]^{2}}{c(c-1)} \text { (nonlinear model) } \\
& =R \text { (linear model). }
\end{aligned}
$$

At early times during nonlinear diffusion, when the normalised expression coefficient $E_{*}(g)$ is everywhere greater than 1, the gradient of the water ratio profile will be smaller than in the linear model. For example, this is apparent in Figure 2a when $R T=0.2$. When the membrane normalised water ratio, $g$ at $\xi=0$, decreases to $c-\sqrt{c(c-1)}$, the value of $\partial g / \partial \xi$, at $\xi=0$, will be the same as that in the linear model. From Figure $2 a$, when $R T=0.35$, the membrane water ratio is already less than the critical value $2-\sqrt{2} \doteq 0.59$, and the nonlinear model predicts $\partial g / \partial \xi$ to be slightly greater than in the linear model. At later times, water depletion at the membrane causes a significant reduction in the expression coefficient and consequently, 


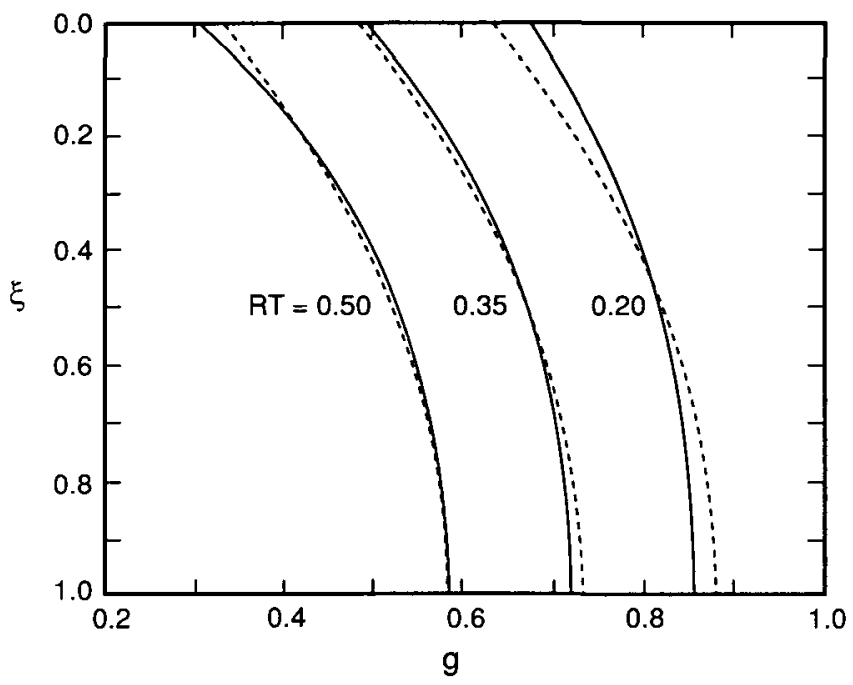

FIGURE 2. (a) Profiles of normalised water ratio $g(\xi, T)$ at times given by $R T=0.2$, 0.35 and 0.5 , during constant-rate expression at dimensionless expression rate $R=0.5$. The full lines represent a weakly nonlinear model $(c=2.0)$ and the dashed lines represent a linear model $(c=\infty)$; (b) As in Figure $2 \mathrm{a}$, but there the full lines represent a moderately model $(c=1.2)$; (c) As in Figure 2a, but there the dimensionless expression rate is lower $(R=0.1)$ and the full lines represent a highly nonlinear model $(c=1.05)$.

constant-rate expression can only be maintained by larger gradients in the water ratio profile. This is evident in the profile of Figure $2 \mathrm{a}$ when $R T=0.5$.

The departure from the linear model will become more pronounced as $c$ approaches 1 . Figure $2 b$ (page 438 ) predicts the water ratio profile in a moderately nonlinear $(c=1.2)$ model material undergoing expression at the same dimensionless rate $R=0.5$ as in Figure 2a. In this case, the gradient $\partial g / \partial \xi$ at $\xi=0$ may first exceed that predicted by the linear model when $g(0, T)$ decreases to $c-\sqrt{c(c-1)} \doteq 0.71$. At later times, the nonlinear model develops water ratio profiles which are much steeper than those of the linear model.

In Figure 2c (page 438), we plot the water ratio profiles of the highly nonlinear model $(c=1.05)$ during expression at a lower dimensionless rate, $R=0.1$. In this case the linear model predicts that $\partial g / \partial \xi \leq 0.1$ for all $\xi$ and all $T$. This is true also in the nonlinear model until $g(0, T) \geq$ $c-\sqrt{c(c-1)} \doteq 0.82$. Up until this time, $g(\xi, T)$ is nowhere far from its mean value $\bar{g}=1-R T$, so that the finite column may be viewed as a small sample and the function $E(g)$ may be estimated from column-averaged values. This uniformity of the water ratio profile has been observed also in a more sophisticated model, analysed numerically by Landman [15]. However, 


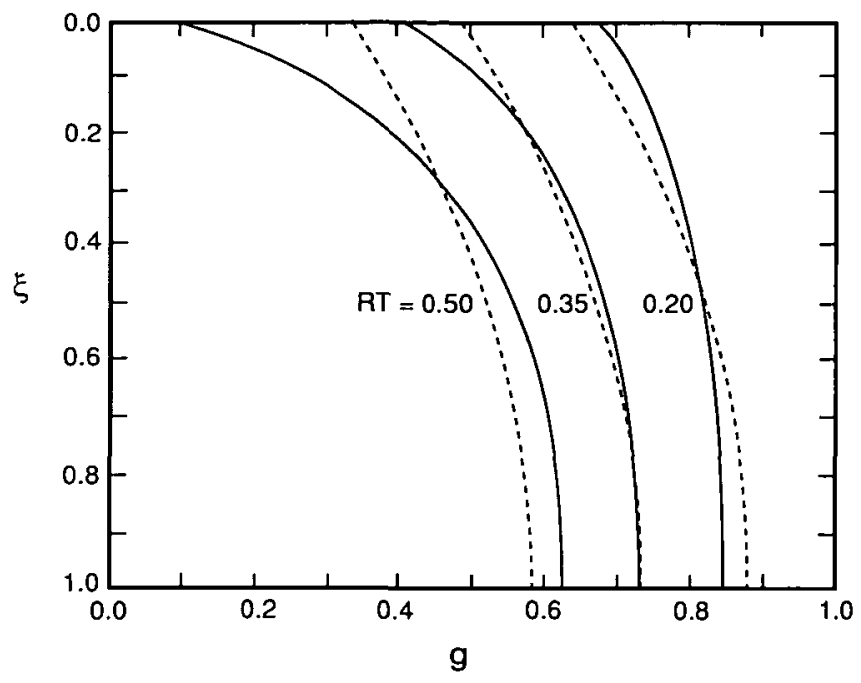

(b)

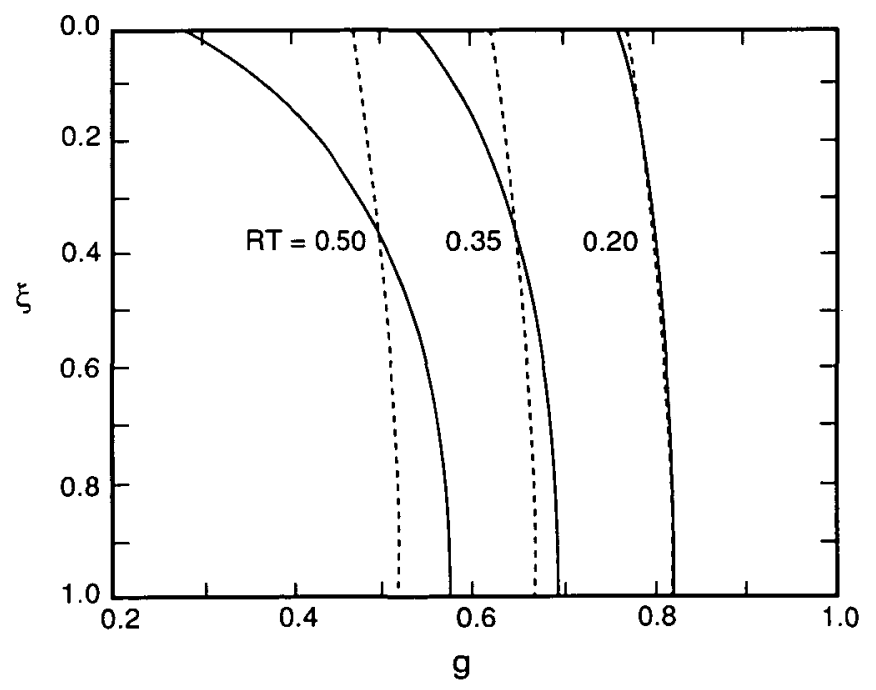

(c) 
even at a low expression rate, at later times, a highly nonlinear model $(c \doteq 1)$ may depart radically from the linear model, as may be seen in Figure $2 \mathrm{c}$ at the last time depicted $(R T=0.5)$.

\section{Water ratio at the boundaries}

As discussed by Banks [1], at any time during expression, the applied external pressure may be deduced from the water ratio at the permeable membrane. Hence, the prediction of water ratio at the membrane is crucial when a prediction of applied pressure is required. The normalised water ratio $g_{m}$ at the membrane may be obtained from the analytic solution by substituting $z=s$ in (A42) and (A41). Hence

$$
g_{m}=c-(c-1) / \mu_{m},
$$

where

$$
\begin{aligned}
\mu_{m}=1 & +\frac{1}{2} \sum_{n=0}^{\infty} \exp (-n(n+1) R / c)\left[\exp \left(-\frac{1}{4}[2(n+1) R / c+s]^{2} / s\right)\right. \\
\times & \left\{-2\left(\frac{s}{\pi}\right)^{1 / 2}+\left(2(n+1)^{2} s+(n+1)[2(n+1) R / c+s]+1\right)\right. \\
\times & f\left(\frac{1}{2} \frac{2(n+1) R / c}{\sqrt{s}}+(n+2) \sqrt{s}\right) \\
& \left.-\left(2 n^{2} s+n[2(n+1) R / c+s]+1\right) f\left(\frac{1}{2} \frac{2(n+1) R / c}{\sqrt{s}}+(n+1) \sqrt{s}\right)\right\} \\
+ & \exp \left(-\frac{1}{4}[2 n R / c-s]^{2} / s\right) \\
\times & \left\{-2\left(\frac{s}{\pi}\right)^{1 / 2}-\left(2 n^{2} s+n[2 n R / c-s]+1\right) f\left(\frac{1}{2} \frac{2 n R / c}{\sqrt{s}}+(n-1) \sqrt{s}\right)\right. \\
& \left.\left.+\left(2(n+1)^{2} s+(n+1)[2 n R / c-s]+1\right) f\left(\frac{1}{2} \frac{2 n R / c}{\sqrt{s}}+n \sqrt{s}\right)\right\}\right],
\end{aligned}
$$

with $s=R^{2} T /[c(c-1)]$ and the function $f$ defined by

$$
f(x)=\exp \left(x^{2}\right) \operatorname{erf} c(x) .
$$

Similarly, the normalised water ratio $g_{p}$ at the piston boundary is obtained by substituting $z=-R / c$ in (A42) and (A41). Therefore,

$$
g_{p}=c-\frac{c-1}{\mu_{p}}
$$


where

$$
\begin{aligned}
\mu_{p}=1+\sum_{n=0}^{\infty} \exp \left(-n(n+1) R / c-\frac{1}{4}[(2 n+1) R / c]^{2} / s\right) \\
\times\left\{-2\left(\frac{s}{\pi}\right)^{1 / 2}+\left(2(n+1)^{2} s+(n+1)(2 n+1) R / c+1\right)\right. \\
\times f\left(\frac{1}{2} \frac{(2 n+1) R / c}{\sqrt{s}}+(n+1) \sqrt{s}\right) \\
\left.-\left(2 n^{2} s+n(2 n+1) R / c+1\right) f\left(\frac{1}{2} \frac{(2 n+1) R / c}{\sqrt{s}}+n \sqrt{s}\right)\right\} .
\end{aligned}
$$

From the exact solution of the linear model [1], the water ratio profile approaches a steady shape in which the normalised membrane water ratio deficit $\bar{g}-g_{m}$ is $R / 3$. The dimensionless time taken for the steady profile to develop is approximately $T=1 / 3$. Thereafter, water depletes at an approximately uniform rate $\partial g / \partial t=-R$, at all locations. For example, in Figure 2a, profiles of the linear model, at times $R T=0.35$ and $R T=0.5$, appear to be successive images of the profile at time $R T=0.2$ under translations

$$
g(\xi, T+0.15 / R)=g(\xi, T)-0.15 .
$$

In contrast, the nonlinear model predicts no such asymptotic approach to a steady profile shape. As the normalised expression coefficient $E_{*}(g)$ decreases, the profile gradient $\partial g / \partial \xi$ must increase if the dimensionless flux $-E_{*} \partial g / \partial \xi$ at the membrane surface, $\xi=0$, is to maintain its prescribed steady value $-R$. This effect may be partially accounted for by scaling down the water ratio deficit by a representative normalised expression coefficient $E_{*}(\bar{g})=c(c-1) /(c-\bar{g})^{2}$, which decreases in time. In Figure 3, we plot $3\left(\bar{g}-g_{m}\right) E_{*}(\bar{g}) / R$ against time, during expression at constant rate $R=0.2$, predicted by the linear model, a moderately nonlinear model $(c=1.15)$ and a strongly nonlinear model $(c=1.05)$. When $R T=0.5$, the strongly nonlinear model has a membrane water deficit $\bar{g}-g_{m}$ approximately five times that of the linear model, but the respective values of the renormalised water ratio deficit $3\left(\bar{g}-g_{m}\right) E_{*}(\bar{g}) / R$ are comparable. At higher expression rates, the value of the membrane water ratio depends more strongly on the degree of nonlinearity of the porous medium.

The value $g=0$ represents a physical lower bound for the water ratio. In practice, the analytic solution for constant-rate expression ceases to be relevant at a maximum time $T_{\max }$ when the analytic model predicts $g\left(0, T_{\max }\right)=0$. We have inverted this equation numerically, to obtain $T_{\max }$ 


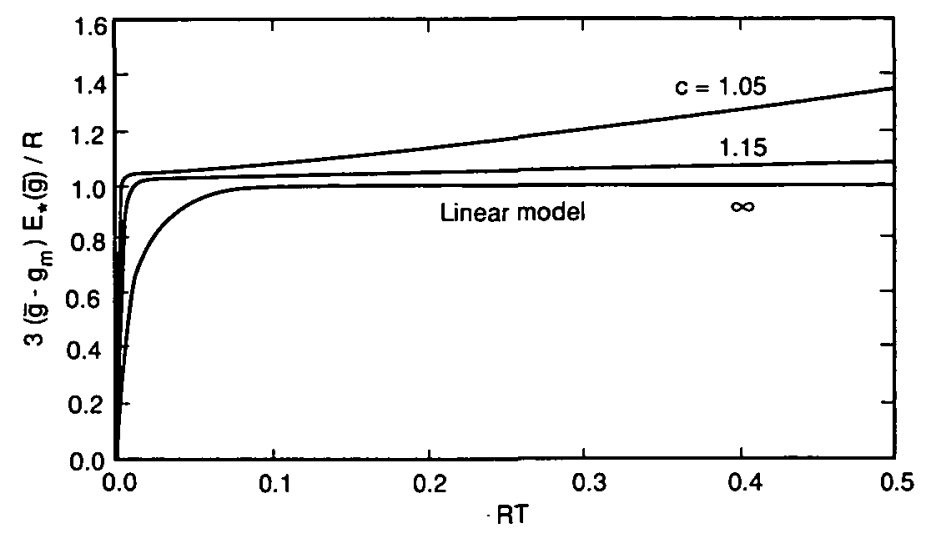

Figure 3. Normalised membrane water ratio deficit $3\left(\bar{g}-g_{m}\right) E_{*}(\bar{g}) / R$ versus time variable $R T$ during expression at constant dimensionless rate $R=0.2$.

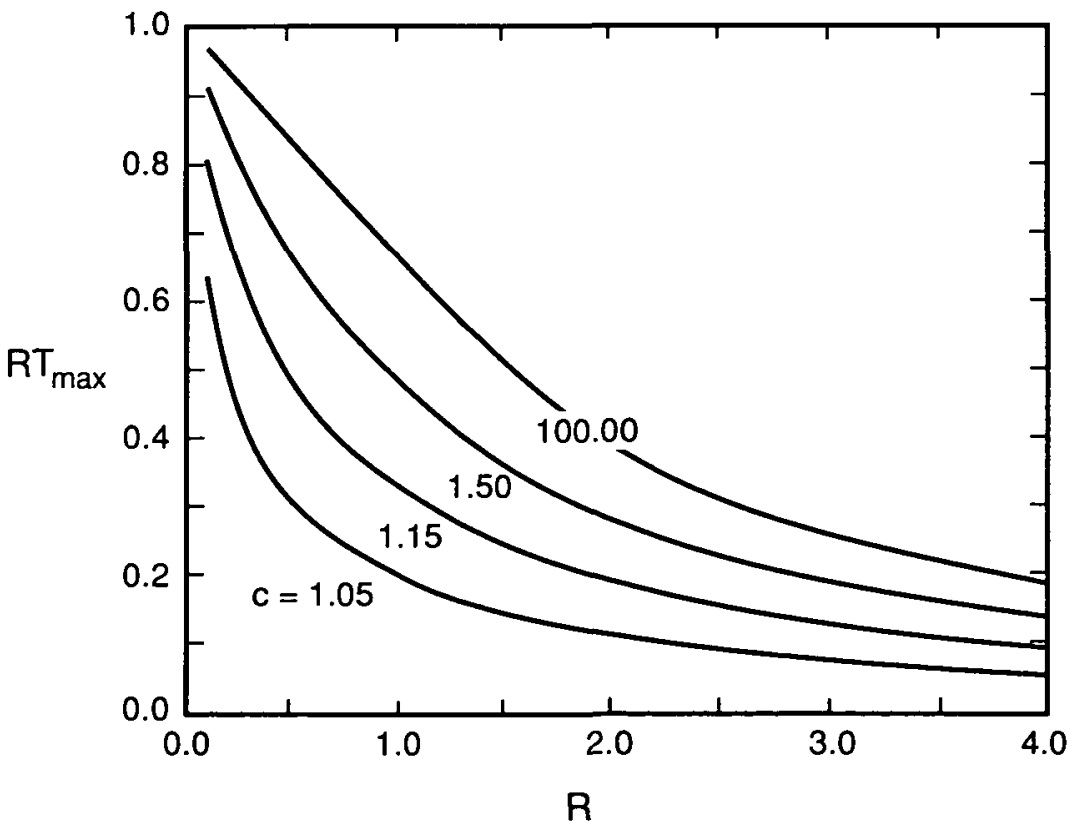

FIGURE 4. The maximum fraction of total initial water volume expressed at constant rate, $R T_{\max }$, is plotted against dimensionless expression rate $R$, for various values of $c$. 
for various values of $R$ and $c$. The product $R T_{\max }$, which approaches 1 as $R$ approaches 0 , is shown in Figure 4 as a function of $R$, for various values of $c$.

\section{Conclusions}

A model expression coefficient $E=\gamma(b-e)^{-2}$, where $e$ is the void ratio and $b$ and $\gamma$ are parameters, has the advantage that the associated nonlinear diffusion equation, representing the flow of liquid during constant-rate expression from a shrinking porous material, admits exact solutions. We have extended previous analytic solutions, to take account of both nonlinearity and finite-column end effects. The effect of nonlinearity has been investigated systematically through the variation of a single nonlinearity parameter $c$. Both the finite-column thickness and the expression rate are incorporated in the definition of the dimensionless expression rate $R$. As $R$ decreases below 0.1 , finite samples of moderately nonlinear materials $(c \geq 1.15)$ show little spatial variation in water ratio and they may be treated, with increasing confidence, as small samples, for example, for the experimental determination of $E(e)$.

Unlike in the linear model, water ratio profiles in the nonlinear model do not approach a constant shape. If $E(e)$ is an increasing function, then at the membrane surface, the gradient of water ratio must increase, in order to maintain the prescribed expression rate.

The analytic solution predicts the membrane water ratio explicitly as a function of time. This offers a computational advantage over numerical solution schemes, in which, even to estimate the water ratio at one location, iterative solution over a large grid is required at many earlier times. The large departure of the membrane water deficit from the value predicted by the linear model, may be approximately compensated for by multiplying by the dimensionless expression coefficient, evaluated at the column-averaged water ratio. However, at very high dimensionless extraction rates of the or$\operatorname{der} R=0.5$, this compensation is not enough. At such high extraction rates, the value of water ratio, at the membrane surface, depends very sensitively on the degree of nonlinearity of the material.

\section{Acknowledgements}

We wish to thank S. Hancy and J. Guth for assistance in numerical evaluations. 


\section{Appendix: Solution of the nonlinear boundary-value problem}

We are required to solve

$$
\partial g / \partial T=\partial / \partial \xi\left[D_{*}(g) \frac{\partial g}{\partial \xi}\right],
$$

with

$$
D_{*}(g)=c(c-1) /(c-g)^{2},
$$

subject to initial conditions

$$
g=1, \quad 0 \leq \xi \leq 1, \quad T=0
$$

and flux boundary conditions

$$
\partial g / \partial \xi=0, \quad \xi=1, \quad 0<T<R^{-1}
$$

and

$$
D_{*} \partial g / \partial \xi=R, \quad \xi=0, \quad 0<T<R^{-1} .
$$

We define a new set of coordinates

$$
\begin{aligned}
& z=\frac{R}{c-1}\left[-\xi+\frac{R T}{c}+\frac{1}{c} \int_{0}^{\xi} g\left(\xi^{\prime}, T\right) d \xi^{\prime}\right], \\
& s=R^{2} T / c(c-1) .
\end{aligned}
$$

Apart from an additional linear transformation, (A6) is the Storm transformation [21], previously applied to nonlinear diffusion problems by Knight and Philip [14]. From (A1) and (A5), it follows that

$$
d s=\frac{R^{2}}{c(c-1)} d T
$$

and

$$
d z=\frac{R}{c} \frac{c-g}{c-1} d \xi-f d T
$$

where

$$
f=\frac{-R}{(c-g)^{2}} \frac{\partial g}{\partial \xi} .
$$

With a change of independent variables $(\xi, T) \rightarrow(z, s)$, derivatives transform as

$$
\frac{\partial}{\partial \xi}=\frac{-R}{c} \frac{c-g}{c-1} \frac{\partial}{\partial z}
$$

and

$$
\frac{\partial}{\partial T}=-f \frac{\partial}{\partial z}+\frac{R^{2}}{c(c-1)} \frac{\partial}{\partial s}
$$


We now define a new dependent variable,

$$
\begin{aligned}
\mu & =\frac{c-1}{c-g} \\
& =\frac{1}{c} \int_{0}^{g} D_{*}(g) d g-\frac{c-1}{c} .
\end{aligned}
$$

Apart from an additional linear transformation, (A11) is the Kirchhoff transformation, which has been applied to nonlinear diffusion problems since the last century [13]. Then (A1) is equivalent to the linear diffusion equation

$$
\frac{\partial \mu}{\partial s}=\frac{\partial^{2} \mu}{\partial z^{2}}
$$

The initial and boundary conditions (A3-A5) become

$$
\begin{gathered}
\mu=1, \quad s=0 \\
\frac{\partial \mu}{\partial z}=0, \quad z=-R / c \\
-\mu^{-1} \frac{\partial \mu}{\partial z}=1, \quad z=s .
\end{gathered}
$$

Hence, the nonlinear boundary value problem (A1-A15) has been transformed to a linear diffusion problem (A12-A15). Cannon [7] shows how to establish uniqueness of its classical solution. The condition (A5) of constantrate expression has been transformed to a radiation boundary condition (A15). If the nonlinear diffusion equation (A1) has an additional nonlinear convection term of the form $\left[\lambda_{1}(c-g)^{-2}+\lambda_{2}\right] \partial g / \partial \xi$, with $\lambda_{1}$ and $\lambda_{2}$ constant, then the linear diffusion equation (A12) would be replaced by Burgers' equation (for example [6]). The latter may be transformed to the linear diffusion equation by an additional Hopf-Cole transformation $[8,11]$, $\mu \rightarrow \phi$, which is essentially equation 2.8 of King [12]. However, following this extra transformation, $\phi$ would satisfy a concentration boundary condition rather than a radiation boundary condition. Hence, in the nonlinear diffusion-convection problem solved by King [12] and generalised by Broadbridge et al. [5], the equivalent linear diffusion problem is essentially different from that addressed here in (A12)-(A15).

Now the Laplace transform $\tilde{\mu}(z, p)$ of $\mu(z, s)$ satisfies $p \tilde{\mu}-1=\frac{\partial^{2} \tilde{\mu}}{\partial z^{2}}$ with general solution

$$
\tilde{\mu}=A e^{-p^{\frac{1}{2} z}}+B e^{p^{\frac{1}{2} z}}+1 / p
$$

The piston boundary condition (A14) implies

$$
B=A e^{2 p^{\frac{1}{2}} R / c} \text {. }
$$


However, the membrane boundary condition (A15) cannot be expressed directly in terms of the variable $p$. Therefore, following King [12], we introduce a moving coordinate

$$
y=z-s
$$

which is fixed at the membrane $y=0$.

Let $\eta(y, x)=\mu(z, s)$. Equation (A12) is equivalent to

$$
\frac{\partial \eta}{\partial s}-\frac{\partial \eta}{\partial y}=\frac{\partial^{2} \eta}{\partial y^{2}}
$$

with initial condition

$$
\eta=1, s=0,
$$

and membrane boundary condition

$$
\eta^{-1} \frac{\partial \eta}{\partial y}=-1, y=0
$$

The Laplace transform $\tilde{\eta}(y, p)$ of $\tilde{\eta}(y, s)$ satisfies

$$
p \tilde{\eta}-1-\frac{\partial \tilde{\eta}}{\partial y}=\frac{\partial^{2} \tilde{\eta}}{\partial y^{2}}
$$

with the general solution

$$
\tilde{\eta}=\frac{1}{p}+C e^{\left[-\frac{1}{2}-\sqrt{p+\frac{1}{4}}\right] y}+E e^{\left[-\frac{1}{2}+\sqrt{p+\frac{1}{4}}\right] y}
$$

Now we apply a Laplace transform boost to represent (A16) in the moving coordinate system $(y, s)$. From Theorem 2 of King [12], the result is

$$
\begin{aligned}
\tilde{\eta}=[1+ & \left.\frac{1}{2} / \sqrt{p+\frac{1}{4}}\right] A\left(\frac{1}{2}+\sqrt{p+\frac{1}{4}}\right) \exp \left(-\left[\frac{1}{2}+\sqrt{p+\frac{1}{4}}\right] y\right) \\
& +\left[1-\frac{1}{2} / \sqrt{p+\frac{1}{4}}\right] B\left(-\frac{1}{2}+\sqrt{p+\frac{1}{4}}\right) \exp \left(\left[\frac{1}{2}+\sqrt{p+\frac{1}{4}}\right] y\right) \\
& +1 / p .
\end{aligned}
$$

The coefficients $A$ and $B$ may be regarded as functions of $p^{1 / 2}$. By comparing (A21) and (A22), we obtain

$$
C=\left[1+\frac{1}{2} / \sqrt{p+\frac{1}{4}}\right] A\left(\frac{1}{2}+\sqrt{p+\frac{1}{4}}\right)
$$

and

$$
E=\left[1-\frac{1}{2} / \sqrt{p+\frac{1}{4}}\right] B\left(-\frac{1}{2}+\sqrt{p+\frac{1}{4}}\right)
$$


Now the boundary condition (A20) may be implemented as

$$
\partial \tilde{\eta} / \partial y=-\tilde{\eta}, \quad y=0 .
$$

Assuming (A21), (A24) implies

$$
\left[\frac{1}{2}-\sqrt{p+\frac{1}{4}}\right] C+\left[\frac{1}{2}+\sqrt{p+\frac{1}{4}}\right] E=-\frac{1}{p} .
$$

Combining (A23) and (A25), we obtain

$$
A\left(\frac{1}{2}+\sqrt{p+\frac{1}{4}}\right)-B\left(-\frac{1}{2}+\sqrt{p+\frac{1}{4}}\right)=\frac{\sqrt{p+\frac{1}{4}}}{p^{2}}
$$

The, by substituting (A17) in (A26), we obtain

$$
\begin{aligned}
A\left(\frac{1}{2}+\sqrt{p+\frac{1}{4}}\right)= & A\left(-\frac{1}{2}+\sqrt{p+\frac{1}{4}}\right) \exp \left(2 \frac{R}{c}\left[-\frac{1}{2}+\sqrt{p+\frac{1}{4}}\right]\right) \\
& +\sqrt{p+\frac{1}{4}}
\end{aligned}
$$

That is,

$$
A(\nu)=A(\nu-1) \exp \left(2 \frac{R}{c}[\nu-1]\right)+\frac{1}{2}[\nu-1]^{-2}-\frac{1}{2} \nu^{-2} .
$$

where $\nu=\frac{1}{2}+\sqrt{p+\frac{1}{4}}$.

Since (A28) is a linear difference equation, $A(\nu)$ may be expressed as a linear superposition

$$
A(\nu)=\frac{1}{2} A_{1}(\nu)-\frac{1}{2} A_{0}(\nu),
$$

where $A_{\rho}(\nu)(\rho=0,1)$ satisfies

$$
A_{\rho}(\nu)=A_{\rho}(\nu-1) \exp \left(2 \frac{R}{c}[\nu-1]\right)+(\nu-\rho)^{-2} .
$$

By analogy with King's ansatz [12], we assume initially

$$
A_{\rho}(\nu)=\sum_{n=0}^{\infty}(\nu-n-\rho)^{-2} \exp \left(\frac{R}{c}\left[f_{n} \nu+h_{n}\right]\right) .
$$

Substitution of (A31) into (A30) leads to the recurrence relations $f_{0}=0 ; \quad f_{m}=f_{m-1}+2 \quad(m \geq 1)$ and $h_{0}=0 ; \quad h_{m-1}-f_{m-1}-2=h_{m}$.

The solution to (A32) is

$$
f_{m}=2 m \text { and } h_{m}=-m(m+1) .
$$


From (A29), (A31) and (A33), a particular solution to the inhomogeneous equation $(\mathrm{A} 28)$ is

$$
A=A^{(p)}(\nu)=\frac{1}{2} \sum_{n=0}^{\infty}\left\{[\nu-(n+1)]^{-2}-[\nu-n]^{-2}\right\} \exp \left(\frac{R}{c}[2 n \nu-n(n+1)]\right) .
$$

However, in this application, King's ansatz does not immediately lead to an applicable solution to our initial boundary-value problem (A12-A15). To the particular solution $A^{(p)}(\nu)$, we may add any solution of the homogeneous equation

$$
A^{(h)}(\nu)=e^{2(\nu-1) R / c} A^{(h)}(\nu-1),
$$

which, in fact, has an infinite-dimensional solution space [16]. We try

$$
A^{(h)}(\nu)=\sum_{n=-\infty}^{\infty} w_{n}(\nu) \exp \left(\frac{R}{c}[2 n \nu-n(n+1)]\right) .
$$

Substitution of (A36) in (A35) leads to the general solution

$$
A^{(h)}(\nu)=\sum_{n=-\infty}^{\infty} w(\nu-n) \exp \left(\frac{R}{c}[2 n \nu-n(n+1)]\right),
$$

with $w$ an arbitrary function.

In the above expression for $A^{(h)}$, we require a function $w$ so that $\tilde{\mu}(z, p)$ given by (A16) and (A17), with $A=A^{(p)}+A^{(h)}$, has an inverse Laplace transform $\mu(z, s)$ which is continuous on the time domain $s \in[0, \infty)$. An appropriate choice is

$$
w(\nu-n)=\frac{1}{2}(\nu-n)^{-2}-\frac{1}{2}(\nu-n-1)^{-2} .
$$

We then obtain

$$
\begin{aligned}
A(\nu) & =A^{(p)}(\nu)+A^{(h)}(\nu) \\
& =\frac{1}{2} \sum_{n=0}^{\infty}\left\{[\nu+n+1]^{-2}-[\nu+n]^{-2}\right\} \exp \left(\frac{R}{c}[-2(n+1) \nu-n(n+1)]\right) .
\end{aligned}
$$

Hence, from (A16) and (A17),

$$
\begin{aligned}
\tilde{\mu}=\frac{1}{p}+\frac{1}{2} \sum_{n=0}^{\infty} e^{-n(n+1) R / c}\left\{\left[p^{1 / 2}+n+1\right]^{-2}-\left[p^{\frac{1}{2}}+n\right]^{-2}\right\} \\
\times\left\{e^{-p^{\frac{1}{2}}[2(n+1) R / c+z]}+e^{-p^{\frac{1}{2}}[2 n R / c-z]}\right\} .
\end{aligned}
$$


From tabulated inverse Laplace transforms (e.g. Oberhettinger and Badii [18]), we obtain

$$
\begin{aligned}
\mu=1+ & \frac{1}{2} \sum_{n=0}^{\infty} \exp (-n(n+1) R / c) \\
\times & {\left[\exp \left(-\frac{1}{4}[2(n+1) R / c+z]^{2} / s\right)\right.} \\
\times & \left\{-2\left(\frac{s}{\pi}\right)^{\frac{1}{2}}+\left\{2(n+1)^{2} s+(n+1)[2(n+1) R / c+z]+1\right\}\right. \\
\times & f\left(\frac{1}{2} \frac{2(n+1) R / c+z}{\sqrt{s}}+(n+1) \sqrt{s}\right) \\
- & \left.\left\{2 n^{2} s+n[2(n+1) R / c+z]+1\right\} f\left(\frac{1}{2} \frac{2(n+1) R / c+z}{\sqrt{s}}+n \sqrt{s}\right)\right\} \\
+ & \exp \left(-\frac{1}{4}[2 n R / c-z]^{2} / s\right) \\
\times & \left\{-2\left(\frac{s}{\pi}\right)^{\frac{1}{2}}-\left\{2 n^{2} s+n[2 n R / c-z]+1\right\}\right. \\
\times & f\left(\frac{1}{2} \frac{2 n R / c-z}{\sqrt{s}}+n \sqrt{s}\right) \\
+ & {\left.\left.\left[2(n+1)^{2} s+(n+1)[2 n R / c-z]+1\right\} f\left(\frac{1}{2} \frac{2 n R / c-z}{\sqrt{s}}+(n+1) \sqrt{s}\right)\right\}\right] }
\end{aligned}
$$

with the function $f$ defined by $f(x)=\exp \left(x^{2}\right) \operatorname{erfc}(x)$.

Equation (A41) gives $\mu$ as a function of $z$ and $s$. Therefore, from (A10), we obtain $g$ as a function of $z$ and $s$,

$$
g(z, s)=c-\frac{c-1}{\mu(z, s)} .
$$

To obtain an exact parametric solution to the problem (A1-A5), we also require $\xi$ as a function of $z$ and $s$. From (A7) and (A10), it follows that

$$
d \xi=\frac{-c}{R}\left[\mu d z+\frac{\partial \mu}{\partial z} d s\right] .
$$

From (A12), (A43) is an exact differential equation, which integrates to

$$
\xi=\frac{-c}{R} \int_{-R / c}^{z} \mu\left(z^{\prime}, s\right) d z^{\prime}+G(s)
$$

for some function $G(s)$. At the piston boundary, $\xi=1$ and $z=-R / c$. Therefore, $G(s)=1$ (constant) and

$$
\xi(z, s)=1-\frac{c}{R} \int_{-R / c}^{z} \mu\left(z^{\prime}, s\right) d z^{\prime}
$$


Now let $\tilde{\xi}(z, p)$ be the Laplace transform of $\xi(z, s)$. From (A45),

$$
\tilde{\xi}(z, p)=\frac{1}{p}-\frac{c}{R} \int_{-R / c}^{z} \tilde{\mu}\left(z^{\prime}, p\right) d z^{\prime}
$$

Therefore, from (A40),

$$
\begin{gathered}
\tilde{\xi}(z, p)=-\frac{1}{2} \frac{c}{R} \sum_{n=0}^{\infty} e^{-n(n+1) R / c}\left\{p^{-\frac{1}{2}}\left[p^{\frac{1}{2}}+n+1\right]^{-2}-p^{-\frac{1}{2}}\left[p^{\frac{1}{2}}+n\right]^{-2}\right\} \\
\times\left\{e^{-p^{\frac{1}{2}}[2 n R / c-z]}-e^{-p^{\frac{1}{2}}[2(n+1) R / c+z]}\right\} .
\end{gathered}
$$

By taking the inverse Laplace transform of (A47), we obtain

$$
\begin{aligned}
\xi=-\frac{c}{R} z-\frac{c}{2 R} \sum_{n=0}^{\infty} e^{-n(n+1) R / c} & \\
\times & {\left[e^{-\frac{1}{4}[2(n+1) R / c+z]^{2} / s}\{[2(n+1) s+2(n+1) R / c+z]\right.} \\
& \times f\left(\frac{1}{2} \frac{2(n+1) / c+z}{\sqrt{s}}+(n+1) \sqrt{s}\right) \\
\left.-[2 n s+2(n+1) R / c+z] f\left(\frac{1}{2} \frac{2(n+1) R / c+z}{\sqrt{s}}+n \sqrt{s}\right)\right\} & \quad\left[[2 n s+2 n R / c-z] f\left(\frac{1}{2} \frac{2 n R / c-z}{\sqrt{s}}+n \sqrt{s}\right)\right. \\
+e^{-\frac{1}{4}[2 n R / c-z]^{2} / s} & \left.\left.-[2(n+1) s+2 n R / c-z] f\left(\frac{1}{2} \frac{2 n R / c-z}{\sqrt{s}}+(n+1) \sqrt{s}\right)\right\}\right],
\end{aligned}
$$

where $f(x)=\exp \left(x^{2}\right) \operatorname{erfc}(x)$ and $s=R^{2} T / c(c-1)$. Since $f(x) \rightarrow 0$ as $x \rightarrow \infty$ (e.g. Gautschi [10]), the series in (A48) and (A41) converge, by comparison with $\sum_{n} \exp \left(-n^{2} \frac{R}{c}\left[1+\frac{R}{c s}\right]\right)$.

An exact parametric solution to the problem (A1-A5) is provided by (A41), (A42) and (A48).

\section{References}

[1] P. J. Banks, "Theory of constant-rate expression and subsequent relaxation", in Drying '85 (eds. R. Toei and A. S. Mujumdar), (Hemisphere, Washington, DC, 1985) 102-108.

[2] P. J. Banks and D. R.Burton, "Press dewatering of brown coal: Part 1- Exploratory studies", Drying Technol. (N.Y.) 7 (1989) 443-475.

[3] P. Broadbridge, "Integrable flow equations that incorporate spatial heterogeneity", Transp. Porous Media 2 (1987) 129-144.

[4] P. Broadbridge, "Infiltration in saturated swelling soils and slurries: exact solutions for constant supply rate", Soil Sci. 149 (1990) 13-22. 
[5] P. Broadbridge, J. H. Knight and C. Rogers, "Constant rate rainfall infiltration in a bounded profile: solutions of a nonlinear model", Soil Sci. Soc. Am. J. 52 (1988) 15261533.

[6] P. Broadbridge and 1. White, "Constant rate rainfall infiltration: A versatile nonlinear model: 1. Analytic solution”, Water Resour. Res. 24 (1988) 145-154.

[7] J. R. Cannon, The one-dimensional heat equation, (Addison-Wesley, Reading, Mass., 1984).

[8] J. D. Cole, “On a quasi-linear parabolic equation occurring in aerodynamics”, Q. Appl. Math. 9 (1951) 225-236.

[9] H. Fujita, "The exact pattern of a concentration-dependent diffusion in a semi-infinite medium: 2", Textile Res. J. 22 (1952) 823-827.

[10] W. Gautschi, "Error function and Fresnel integrals", in Pocketbook of Mathematical Functions (eds. M. Abramowitz and I. A. Stegun), (Verlag Harri Deutsch, Frankfurt, West Germany, 1964).

[11] E. Hopf, "The partial differential equation $u_{t}+u u_{x}=\mu u_{x x}$ ", Commun. Pure Appl. Math. 3 (1950) 201-230.

[12] M. J. King, "Immiscible two-phase flow in a porous medium: utilization of a Laplace transform boost", J. Math. Phys. 26 (1985) 870-877.

[13] G. Kirchhoff, Vorlesungen über die Theorie der Warme (Barth, Leipzig, 1894).

[14] J. H. Knight and J. R. Philip, "Exact solutions in nonlinear diffusion", J. Eng. Math. 8 (1974) 219-227.

[15] K. A. Landman, "Some moving boundary problems in solid/liquid separation", in Proceedings of the Mini-Conference on Free and Moving Boundary and Diffusion Problems (eds. J. Hill and R. Anderssen), (Centre for Mathematical Analysis, Australian National University, Canberra, 1990) to appear.

[16] L. M. Milne-Thomson, The calculus of finite differences (Macmillan, London, 1933).

[17] T. Murase, M. Iwata, P. J. Banks, I. Kato, N. Hayashi and M. Shirato, "Press dewatering of brown coal: Part 2-Batch and continuous-screw operations", Drying Technol. (N.Y.) 7 (1989) 697-721.

[18] F. Oberhettinger and L. Badii, Tables of Laplace transforms (Springer, Berlin, 1973).

[19] J. R. Philip, “The theory of infiltration: 4. Sorptivity and algebraic infiltration equations", Soil Sci. 84 (1957) 257-264.

[20] D. E. Smiles, "Constant rate filtration of bentonite", Chem. Eng. Sci. 33 (1978) 13551361.

[21] M. L. Storm, "Heat conduction in simple metals", J. Appl. Phys. 22 (1951) 940-951. 